\title{
STRÖMGREN PHOTOMETRY OF STARS IN BAADE'S WINDOW
}

\author{
ALEX RUELAS-MAYORGA AND GABRIEL GARCíA-RUíZ \\ Instituto de Astronomía. UNAM \\ Cd. Universitaria. Apartado Postal 70-264 \\ C.P. 04510. México, D.F. México \\ rarm@astroscu.unam.mx
}

\section{Abstract}

Baade's Window (BW) has long been observed because of its low absorption and its position near the galactic centre. These characteristics permit the observation of many stars which are presumably members of the galactic bulge; although it is not possible to exclude contamination of this field by stars belonging to the galactic disc. Geometric arguments as well as galactic models allow to see that most of the stars are located far away from the solar neighbourhood. Based on this fact alone, many astronomers have assumed that the entire stellar population observed in BW consists of bulge stars.

We present uvby photometry of 232 stars in BW obtained with a fourchannel spectrograph-photometer, which uses exit slots and optical interference filters to define the bandpasses; the edges of the exit slots fall at wavelenths where the filter transmissions are low, generally about $15 \%$.

The results are very preliminary and are presented in the form of diagrams; due to the lack of space, they shall be presented elsewhere. However, our main conclusions are as follows: i) In the HR diagrams ( $v$ vs $(b-y)$ and $y$ vs $(b-y))$ it is possible to separate the stellar population observed in two dominant branches which may consistently be associated with the disc and the bulge populations, ii) Metallicity values derived from the $\left[m_{1}\right]$ index show two dominant groups, one with high and another one with low metallicities which could very well be ascribed to the disc and bulge populations in BW, and finally; iii) the value of the metallicity decreases as the brightness of the stars decreases, in good agreement with results published earlier. 